\title{
Measurement of urine output by weighing nappies
}

\section{S Oddie, R Adappa, J Wyllie}

The effect of humidity on measurement of neonatal urine output was assessed by weighing nappies in a clinically relevant context. Saline was used as dummy urine, on modern nappies in incubators at various humidity settings. In at least some additional humidity, no clinically relevant evaporative loss occurred.

M easurement of urine output is an important element of neonatal intensive care. Urethral catheterisation may aid urine collection and measurement but may be complicated by catheter bypassing or dislodgement, and carries a risk of introducing bacterial sepsis. ${ }^{1}$ Measuring urine output by weighing nappies before and after a period of nursing care is a widely used technique in the United Kingdom. Disadvantages of this technique include the possibility of stool contamination of the nappy, which may make estimation of urine output difficult. In addition, and depending on the type of nappy used, evaporative losses of urine may potentially lead to underestimation of urine output. Cooke et $\mathrm{al}^{2}$ considered this, and recommended that where this technique was used nappies should be weighed every two hours.

In our unit we usually assess urine output by weighing nappies. We investigated the effect of environmental humidity on evaporative weight change of nappies.

\section{METHODS}

We used a Draeger 8000 IC incubator left at steady state humidity and temperature settings for at least one hour before starting experiments. We set the incubator to deliver either no additional humidity or humidity at settings of $40 \%$, $60 \%$ or $85 \%$. We set the incubator temperature at $39^{\circ} \mathrm{C}$. We added a $5 \mathrm{ml}$ quantity of $0.9 \% \mathrm{NaCl}$ to preweighed nappies (Moltex Baby Hygiene, Mayen, Germany), approximate weight $17 \mathrm{~g}$, and reweighed each one. We then placed batches of six nappies in the incubator, open with the absorbant side up. Nappies were weighed between three and six times in each experiment over about six hours. We have included data on 57 nappies here. We used a Mettler PC 440 precision balance accurate to $0.001 \mathrm{~g}$ for all weight measurements. To enable precise estimates of the degree of weight change over differing experimental periods, we calculated rates of weight change for each nappy. Analysis therefore assumes linear weight loss over time. We made statistical comparisons by analysis of variance of rates of change of nappy weight using Statview version 5.

\section{RESULTS}

Significant differences $(\mathrm{p}<0.05)$ exist between the rates of weight change for each humidity setting (fig 1).

\section{CONCLUSIONS}

No nappy kept in $85 \%$ humidity lost weight. Nappies kept in $40 \%$ humidity had variable weight changes. Only nappies nursed in no additional humidity had evaporative weight loss that may lead to clinically significant underestimation of urine output. Such nappies may lose $2.3 \mathrm{~g}$ of a $5 \mathrm{~g}$ urine weight over a typical nursing period of six hours.

Our study may be criticised for the assumption of linear weight change over time. This is based on data such as that in the figure in the paper of Cooke et al. ${ }^{2}$ However, the nappies in different humidity settings were studied over similar periods. Therefore even had weight change not been linear, our results remain informative.

Our study suggests that the technique of estimating urine output by weighing nappies is robust provided that infants are nursed in some additional humidity. Our findings are at variance with others in the literature ${ }^{23}$ insofar as they suggest that this technique may be used over a six hour period without important loss of fidelity.

Our findings are most relevant for extremely preterm infants, with low urine outputs. For this reason we concentrated on a $5 \mathrm{ml}$ volume of dummy urine. We avoided using human or animal urine to minimise hazard to infants later using the same incubators and to maintain consistency of tonicity over several experiments.

Other studies have found significant evaporative losses from nappies nursed in this way. ${ }^{2}{ }^{3}$ We therefore chose to perform these experiments in an incubator at $39^{\circ} \mathrm{C}$ to maximise any evaporative losses that we might encounter. Differences in environmental humidity through local climate or use of air conditioning in their institutions may explain some of the differences between our findings and those of other workers. We do not have air conditioning in our unit. Environmental humidity varies, but was measured using a wet bulb hygrometer at $28 \%$ on one occasion.

Other authors have shown that the type of nappy significantly affects the rate of evaporative loss. We chose

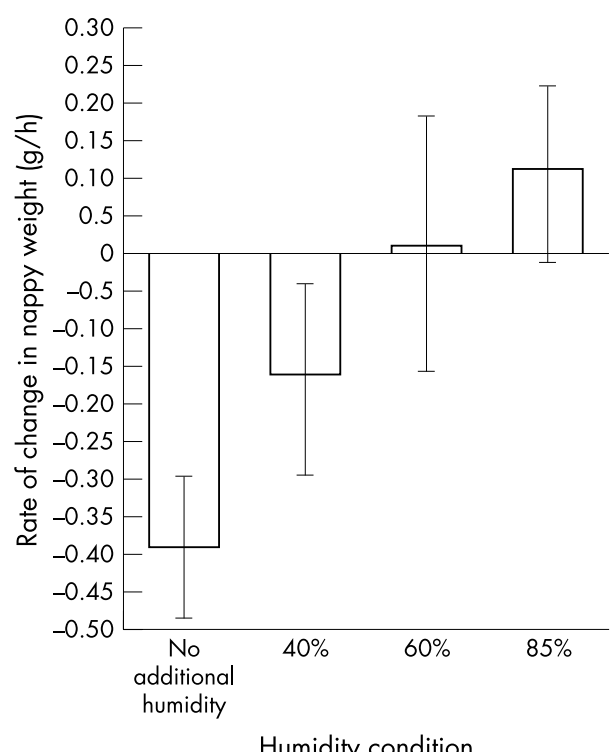

Figure 1 Rate of change in nappy weight in relation to humidity. The $95 \%$ confidence intervals are shown. 
to use Moltex nappies for this series of experiments. These nappies contain naturally absorbent fibres and hydrophilic gel. They are marketed as being highly absorbent and are in routine use on our unit.

One practical consequence of this study is that we have bought new scales (weighing in increments of $1 \mathrm{~g}$ to replace scales weighing in increments of $5 \mathrm{~g}$ ) for weighing nappies to assess urine output.

\section{Authors' affiliations}

S Oddie, R Adappa, J Wyllie, Neonatal Unit, James Cook University Hospital, Middlesbrough TS4 3BW, UK
Correspondence to: Dr Oddie, Neonatal Unit, James Cook University Hospital, Marton Road, Middlesbrough TS4 3BW, UK

Accepted 2 December 2002

\section{REFERENCES}

1 Foxman B. Epidemiology of urinary tract infections: incidence, morbidity, and economic costs. Am J Med 2002;113(suppl 1A):5S-13S.

2 Cooke RJ, Werkman S, Watson D. Urine output measurement in premature infants. Pediatrics 1989;83:116-18.

3 Gouyon JB, Sonveau N, d'Athis P, et al. Accuracy of urine output measurement with regular disposable nappies. Pediatr Nephrol 1994;8:88-90. 